\title{
Neuropsychobiology 1987;18:219
}

\section{Author Index}

Al-Nakib, W. 144 Ansseau, M. 77 Arnetoli, G. 149 Arqué, J.M. 127 Åsberg, M. 89 August, G.J. 105

Battagia, A. 149 Bellodi, L. 68 Belon, J.P. 21 Brambilla, F. 68 Brancato, V. 5, 68 Brockington, K. 57

Campbell, S.S. 169 Carey, R.J. 199 Cassano, G.B. 74 Castillo, A. 189 Catalano, M. 5 Christiansen, K. 21 Cohen, C. 1 Cohen, S. 9 Collins, A. 83 Coppola, R. 97 Coyle, K.B. 144 Deistler, M. 155 Depoortere, H. 160 Dimpfel, W. 212 Donovan, C.B. 144 Dreux, C. 1

Edman, G. 89

Facchinetti, F. 68 Ferber, G. 43 
Fiet, J. 1

Frenckell, R. von 77 Friedman, R. 43

Gaillard, J.-M. 60 Gasperini, M. 5 Gaudel, G. 21 Genazzani, A.R. 68 Giannaccini, G. 74 Gillin, J.C, 169 Gimeno, V. 110 Goetze, U. 175 Grignani, A. 149 Guéchot, J. 1 Hagnell, O. 13 Herrmann, W.M. 97 Higgins, P.G. 144 Higuchi, T. 185 Hommer, D.W. 113

Jacqmin, P. 77

Kammen, D.P. van 113 Kenney, S. 199 Khan, A. 9 Klieser, E. 122 Klinteberg, B. af 89 Knussmann, R. 27 Koch, R. 212 Komoda, T. 185 Krebs, E. 43 Krijzer, F.N.C.M. 51 Lanke, J. 13 Lee, KL! 205 
Lehmann, E. 122 Lehmkuhl, P. 37 Lempérière, T. 1 Leonard, B.E. 118 Lépine, J.P. 1 Lifshitz, K. 205 Lucacchini, A. 74 Lucca, A. 5

Malas, K.L. 113 Malmgren, R. 83 Marazziti, D. 74 Mariategui, J. 189 Martin, P. 21 Martini, C. 74 Massol, J. 21 Matejcek, M. 43 Miura, M. 185 Molen, R. van der 51 Nijssen, A. 195 Nilsson, C.-G. 83

O’Connor, W.T. 118 Öjesjö, L. 13 Oreland, L. 89

Paganini, M. 149 Perugi, G. 74 Petraglia, F. 68 Pichlmayr, I. 37 Pietrini, P. 74 Placidi, G.F. 74 Prass, D. 37 Pritchard,|W.S. 105 
Rafhn, Y. 60 Raz, N. 105 Reschenhofer, E. 155 Rorsman, B. 13 Roy,|A. 57 Russell, R.W. 134

Sacchetti, G. 149 Sakagishi, Y. 185 Schalling, D. 89 Schatton, W. 212 Schelvis, P.R. 195 Segura, R. 127 Smeraldi, E. 5,68 Smith,|A.P. 144 Sotillo, C, 189 Soubrié, P. 21 Spüler, M. 212 Sugishita, M. 185 Susswein, S. 205

Tissot, T. 60 Tölle, R. 175 Torrubia, R. 127 Tyrrell, D.A.J. 144

Versari, A. 149 Vollmer, R. 155

Widmer, J. 60 Willman, J.S. 144

Yamauchi, T. 185 Yamazaki, J. 185

Zappoli, R. 149 